\title{
Effectiveness of a new short-stay unit for elective low complexity patients' admissions to improve patient flow
}

\author{
Berta Ortiga $^{1}$, Carlos Bartolome ${ }^{1}$, Xenia Acebes ${ }^{1}$, Mari Fe Viso ${ }^{1}$, Guillem Marca ${ }^{2}$, Alfredo García ${ }^{1}$ \\ 1. Bellvitge University Hospital and Bellvitge Biomedical Research Institute (IDIBELL), University of Barcelona, \\ L'Hospitalet de Llobregat, Barcelona, Spain. 2. Communication and Business Administration Faculty, University of Vic, Vic, \\ Barcelona, Spain.
}

Correspondence: Berta Ortiga. Address: Hospital Universitari de Bellvitge, Subgerència, C. Feixa Llarga s/n, 08907 L'Hospitalet de Llobregat, Barcelona, Spain. E-mail: bertao@bellvitgehospital.cat

Received: October 7, 2013

DOI : $10.5430 /$ jha.v3n3p44

Accepted: December 10, 2013 URL: http://dx.doi.org/10.5430/jha.v3n3p44
Online Published: December 30, 2013

\section{Abstract}

Background/Objective: Specialized care in an acute hospital is the highest resource consuming type of health care. Performance improvement in health care should look for a better application of medical knowledge and resource consuming at the same time as managers locally redesigned processes and organizations in order to be more cost-effective at healthcare services delivery. The aim of this study was to demonstrate the effectiveness of creating a short-stay unit for elective patients as an alternative to the elective patients being admitted in an acute ward.

Methods: We included all elective patients admitted in a university hospital between the 1st of January and the 31st of December 2007, as well as those admitted during the same period of 2009, after two hospital wards transformation into a short-stay unit. We used the Hospital General Database for collecting information on years 2007 and 2009. Main key performance indicators were length of stay, pre-surgery length of stay, rotation rate, discharge planning rate and cost. For statistical bivariate analysis, we used a Chi-squared for linear trend for qualitative variables and a $T$-test and a Wilcoxon signed ranks test and a Mann-Whitney test for non-normal continuous variables. Significance at $p<.05$ was assumed throughout.

Results: We included 10,678 patients, 4,423 during 2007 and 6,255 during 2009. Mean length of stay was 4.3 days (IC 95\%: 4.09-4.51) in 2007 and 2.8 days (IC 95\%: 2.61-3.01) in 2009 ( $p .05$ ). Pre-surgery length of stay was reduced from 0.5 days (IC 95\%: 0.44-0.56) in 2007 to 0.2 days (IC 95\%: 0.17-0.23) in $2009(p<.05)$. The rotation rate was of 92 patients/bed in 2007 and of 126 patients/bed in 2009 ( $p<.05)$. The median number of planned discharges grew from 43.05\% in 2007 to $86.01 \%$ in 2009. Closing two hospital wards at weekends has generated savings of $€ 805,376.32$ through the reduction of 22 nurse employees.

Conclusions: In conclusion, this approach to hospital bed management in a tertiary hospital has proven both possible and efficient. The creation of short-stay units for elective patient admissions allowed an increase in productivity per hospital bed due to a higher rotation rate and a reduction in human resources costs due to closing hospital wards at weekends.

\section{Key words}

Effectiveness, Operations research, Hospital administration 


\section{I ntroduction}

The largest single source of health care costs is hospitals, which account for close to $40 \%$ of all health care expenditure. In recent years, hospitals have engaged in various cost-cutting efforts in order to reduce the number of beds ${ }^{[1,2]}$. In this sense, bed management forms an important part of operational capacity planning and control in a hospital; it can be considered as a wider activity concerned with the efficient use of resources ${ }^{[3]}$.

Although the impact on the medical outcome of a patient's delay for an appropriate bed is difficult to measure and clearly depends upon the specific medical condition, hospitals do recognize the adverse consequences of delays. Moreover, backups in emergency rooms, surgical recovery rooms, and labour/delivery rooms reduces the ability to admit new patients and may result in lost revenues from the hospital either to sending emergency patients to other hospitals ${ }^{[4]}$, or to surgery cancellations and its consequences to waiting lists.

On the other hand, the workload at clinical wards is most often highly variable and less flexible, leading to the need for rational overcapacity to respond to peaks in demand for beds. Previous studies have shown that the variation in the number of scheduled patients admitted is at least as large as the variation in the number of emergency admissions, and it is usually larger. The variability in the number of admissions leads to highly variable bed occupancy ${ }^{[5]}$ and the variation seen in the demand and availability of beds generates Erlang queues that create problems when new patients need to be admitted ${ }^{[6]}$.

In this sense, designing a sustainable flexible healthcare system that it is able to cope with demand variation of healthcare needs should be a major priority of health authorities anywhere. Performance improvement in health care should look for a better application of medical knowledge and resource consuming at the same time as managers locally redesigned processes and organizations in order to be more cost-effective at healthcare services delivery ${ }^{[7]}$. The provision of a better patient experience due to an increase in flexibility and performance improvement is also a key factor to an efficient hospital management and ultimately, to the healthcare system sustainability. The aim of this study was to demonstrate the effectiveness of creating a short-stay unit for elective patients as an alternative to the elective patients being admitted in an acute ward.

\section{Method}

This intervention was performed in an 850-bed university affiliated hospital nearby Barcelona (Spain) and it is a member of the National Health System. It receives more than 90,000 emergency department visits per year and the mean number of elective admissions is 1,650 (95\% CI: 1,609-1,691) per month, excluding day surgery. For this study, we organized an interdisciplinary group integrated by physicians, nursing personnel, officers and patients/families to identify functional bottlenecks and clinical pathways to be improved. The team listed several interventions and we prioritized the high impact changes whose goal consisted in: reducing variation within the admission and discharge processes for elective patients and avoiding last minute surgery cancellations that have an impact on patients' and their relatives' lives as well as in the waiting list management. The multi-faceted intervention was implemented between April and December 2008. For this study, we included all elective patients admitted to two hospital wards before the multi-intervention, between the 1st of January and the 31st of December 2007, and after the implementation, between the 1st of January and the 31st of December 2009. Day surgery patients were not included.

The short-stay unit (SSU) was comprised of two hospital wards and each one had three reception chairs and 22 beds. The three reception chairs were points of care and they were coordinated and organized by nurses who performed the preoperative preparation. In general, patients were admitted to the hospital two hours before surgery. In the admission form there were the physicians' notes describing any special care or provider-order entry needed, as well as the inappropriateness of the preparation in a chair. The Admission Department performed chair assignment and the hospital's bed management. Patients were admitted during the whole day. There was a double nursing staff between 7:00 and 8:00 in 
the morning, as the two nursing shifts had an overlapping hour. That was the time when there was the highest demand and more patients needed surgery preparation, being in a chair or in a SSU bed. General surgery preparation pathways were uploaded to the hospital intranet.

A centralized team, the Admission Department, managed the hospital beds. They had a global vision of the bed occupancy throughout the hospital at any moment, and that allowed an optimal patient allocation of emergency and elective patients, transfers of outlier patients and admission and discharge time monitoring. For patient allocation, chairs were used to prepare patients whose expected length of stay was over 48 hours, while admissions to a SSU bed were for scheduled patients with an expected length of stay between $24 \mathrm{~h}$ and $72 \mathrm{~h}$. SSU beds or the preparation chairs were never used for inpatients coming from the emergency department. Patients admitted to a preparation chair had to be allocated to a hospital bed later in the same day of admission, after surgery and recovery time. In this sense, there was usually enough time to find available beds with the discharges of the day as most patients with longer length of stay needed post-surgery intensive care for at least 24 hours before going to a conventional ward and this fact added time flexibility in finding a free bed. SSU beds and reception chairs were closed during weekends, from Friday evening until Monday 7:00 am. Closing time varied, as it depended on discharges and transfers to the rest of the opened hospital wards. Transferred patients were mainly discharged on Saturday. For SSU patients, discharge time was planned together with the admission. Patients admitted to reception chairs planned discharge at least 24-hour in advance so that everything was ready at the time set.

We used the Hospital General Database for collecting information on patient demographics, main procedure, dates of admission, discharge and of surgery, number of delayed emergency department inpatients due to lack of beds every morning, number of cancelled patients and the reason, number of discharges and how many were planned at least one day in advance, number of patients located out of their medical service and number of day-surgery patients. This multi-faceted intervention did not interfere in the clinical management of the patients and for this reason we did not consider looking for ethical approval. All the interventions were hospital based.

The main outcome variables were: median length of stay, proportion of patients admitted on the same day of surgery, percentage of planned discharges, number of surgery cancellations, proportion of day-surgery, median number of delayed emergency admissions at 8:00 am due to lack of bed, risk-adjusted mortality rate, risk-adjusted complications rate and risk-adjusted readmissions rate and median number of patient outliers. We define patient outliers as those patients admitted to a bed that it is not located in the ward of the medical specialty to which they should be admitted. The intervals of time used in our measurements are days, hours and their fractions. Categorical variables were described using the total number of cases ( $\mathrm{N}$, days) and the percentage of each category. For bivariate analysis we used the Chi-squared for linear trend. Continuous variables were presented as a mean or a median, with their interval of confidence or interquartile range, respectively. Bivariate analysis was performed using a $T$-test or the Wilcoxon signed ranks test or the Mann-Whitney test. Significance at $p<.05$ was assumed throughout. We used Statistical Software Program for Windows (version 14) for statistical analysis.

\section{Results}

The development of a SSU for scheduled patients had consequences to different hospital departments. First, the admission of most of the scheduled patients was now between 7:00 am and 9:30 am and to the SSU-beds or preparation chairs. Hospitalization orders were confirmed with the medical services and also checked with the patients the day before admission, until 3:00 pm. At the same time, the morning before the admission day, all patients were assigned to a SSU bed or to a preparation chair from the surgery reception unit. In this sense, all patients had a specific location 24 hours in advance of their admission to the hospital, which was also introduced in the electronic-health information system, and therefore admission was a planned and safer process. In addition, the discharge day of SSU-patients was planned together with the admission day and also entered in the electronic information system. These working dynamics positively contributed to the standardization of the admission and discharge processes, explained in a previous paper ${ }^{[8]}$. The 
standardization of the discharge process was based on two main concepts: Firstly, the need for planning the activity and secondly, the promotion of a team incorporating different professional groups all working in the hospital. In this sense, the median number of planned discharges grew from 43.05\% in 2007 to $86.01 \%$ in 2009 (see Figure 1).

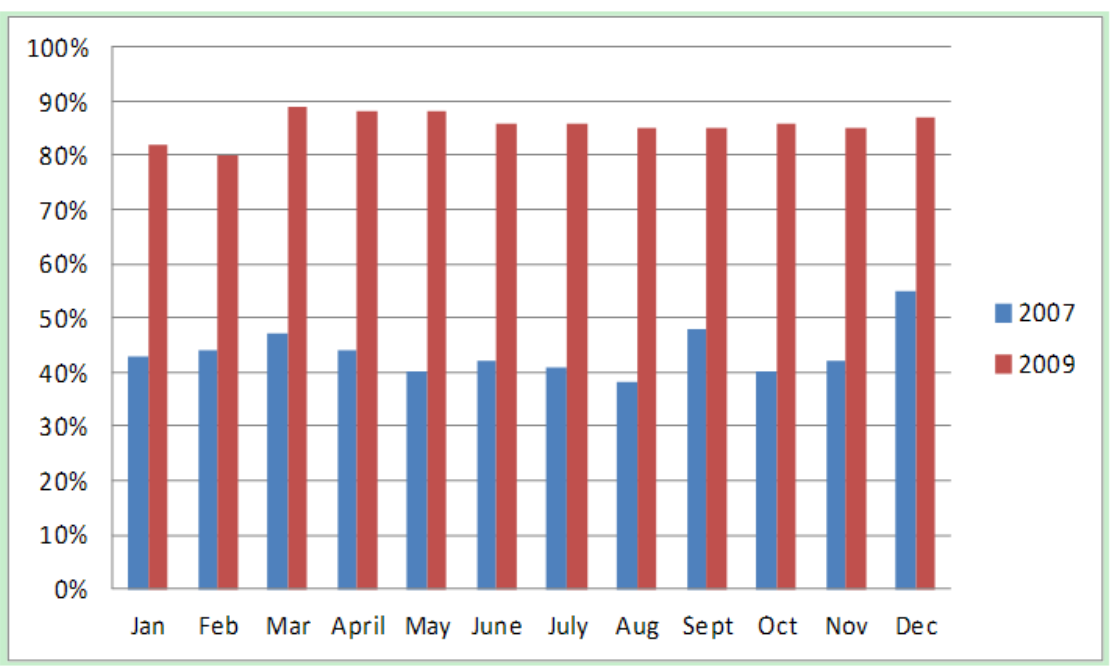

Figure 1. Comparison of percentage of planned discharges during 2007 and 2009, by months

The impact of the SSU to human resources management was high due to the fact that these two wards, together with the surgery reception unit, closed every Friday evening and therefore the number of employees needed, basically nurse personnel, was 50\% less (22 staff less, 11 for each SSU- ward). This meant a cost reduction of €805,376.32 per year (see Table 1). At the same time, nurses changed their working shifts, so that the twelve-hour shift every two days changed for a 7-hour shift every working day in the morning and afternoon, and a 10-hour shift at nights. The fact that most patients had an expected length of stay of two days (see Figure 2) meant that they had the same nurse during all their hospitalization process and this enhanced the patient-nurse relationship and the nurse's role as a patient manager.

Table 1. Revenues and payments of the SSU (including surgery reception unit in one bedroom of each ward)

\begin{tabular}{ll}
\hline Item & Amount \\
\hline Payments & \\
6 chairs for the Surgery Reception Unit & $€ 23,538.54$ \\
Two more showers for patient use & $€ 13,700.00$ \\
Fungible spending from wards & $-€ 36,711.00$ \\
House officer (33\% of house officer for confirming telephone call) & $€ 4,950.00$ \\
Nurse personnel (Reduction of 4.6 auxiliary nurses and 5.7 nurses per ward) & $-€ 805,376.32$ \\
Total Payments & $-€ 799,898.78$ \\
Revenues & \\
Cancelled admissions due to lack of bed (174*3,023€/discharge) & $€ 522,979.00$ \\
Total Revenues & $€ 522,979.00$ \\
Total & $€ 1,322,877.78$ \\
\hline
\end{tabular}

Other costs and revenues due to the transition of a conventional ward to a SSU ward plus reception unit are presented in Table 1. As the SSU wards were closed at weekends, the costs due to fungible spending such as bed clothing, serums, unspecific drugs for inpatients, cleaning personnel, patients' menus, etc. implied a mean estimation savings of $€ 36.711$ for the two wards. In addition, the scheduled admissions of the hospital were mainly done in two hospital wards, and the nurse personnel demanded an extra shower for each SSU ward to avoid the shower to become a potential bottleneck for the patients before going to the surgery theatre. The hospital had to buy six chairs for the expected long-length of stay patients 
preparation which had a cost of $€ 3,923.09$ each. The main revenue of the hospital was that the number of patients that had to be cancelled due to lack of beds drastically decreased in 174 patients.

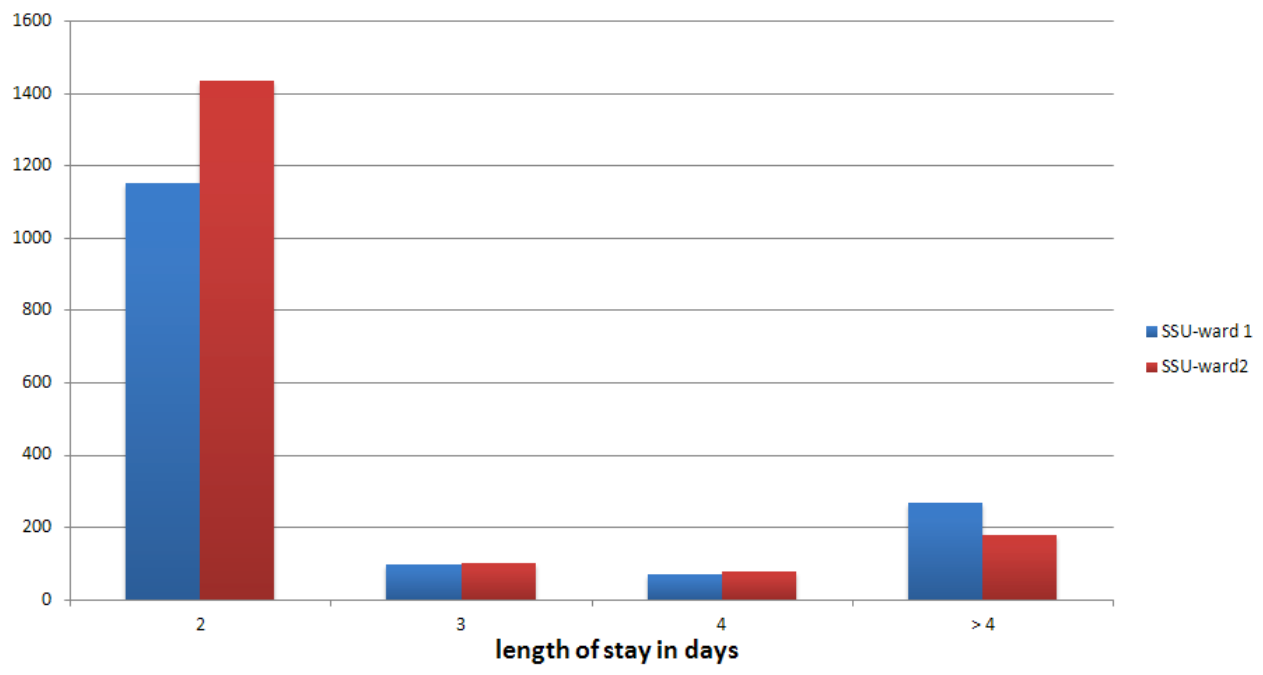

Figure 2. Patients admitted to the two SSU-wards by length of stay (days)

During 2007 and 2009, these two hospital wards admitted 10,678 patients. In 2007, the regular hospital wards admitted 4,423 patients and 6,255 patients in 2009, when they had been transformed into SSU. The mean length of stay was of 4.3 days (95\% IC: 4.09-4.51) in 2007 and of 2.9 days (95\% IC: 2.61-3.01) in $2009(p<.05)$. There was a reduction in the pre-surgery length of stay, due to the "same day of admission as surgery" policy implemented, from 0.5 days (95\% IC: 0.44-0.56) in 2007 to 0.2 days (95\% IC: 0.17-0.23). The rotation index changed from 92 patients/bed in 2007 to 126 patients/bed in 2009, when the two SSU-wards were closed at weekends.

In all the hospital, the median number of patients outliers per day was 70 patients in 2007 and 62 patients in $2009(p<.05)$. The number of surgery cancellations due to lack of beds was reduced from 216 patients in 2007 to 42 patients in 2009 and so did the median number of emergency patients waiting for a bed in the morning, which changed from five patients in 2007 to three patients in $2009(p<.01)$. Other key performance indicators results of the hospital activity and quality of care are showed in Table 2 and in Table 3, respectively.

Table 2. Main key performance indicators during years 2007 and 2009

\begin{tabular}{|c|c|c|c|c|c|}
\hline & 2007 & IQR: Q1-Q3 & 2009 & IQR: Q1-Q3 & $p$ value \\
\hline Same day of surgery admission & $64.87 \%$ & $51.07 \%$ to $70.02 \%$ & $86.01 \%$ & $83.50 \%$ to $88.93 \%$ & $<.05$ \\
\hline Pre-surgery length of stay (days) ${ }^{*}$ & 0.58 & 0.53 to 0.70 & 0.26 & 0.24 to 0.32 & $<.05$ \\
\hline $\begin{array}{l}\text { Length of stay (without day surgery, } \\
\text { days) }{ }^{*}\end{array}$ & 8.56 & 6.88 to 10.01 & 7.93 & 6.78 to 9.51 & .051 \\
\hline $\begin{array}{l}\text { Scheduled patient length of stay (without } \\
\text { day surgery, days) }\end{array}$ & 4.85 & 3.73 to 6.33 & 4.54 & 3.62 to 4.54 & $<.05$ \\
\hline Emergency patients length of stay (days) ${ }^{*}$ & 11.64 & 9.82 to 13.93 & 11.46 & $9.49-13.56$ & .22 \\
\hline Cancelled interventions & 216 & - & 42 & - & - \\
\hline Emergency patients admitted to hospital & $10.46 \%$ & $9.26 \%$ to $11.90 \%$ & $10.49 \%$ & $9.20 \%$ to $12.13 \%$ & .33 \\
\hline Discharge planning & $43.05 \%$ & $40.09 \%$ to $45 \%$ & $86.01 \%$ & $84.92 \%$ to $87.10 \%$ & $<.01$ \\
\hline $\begin{array}{l}\text { Daily patients placed out of service } \\
\text { (outliers) }\end{array}$ & 70 & 56 to 78 & 62 & 49 to 69 & $<.05$ \\
\hline Emergency inpatients waiting for a bed & 5 & 1 to 11 & 3 & 1 to 7.50 & $<.01$ \\
\hline
\end{tabular}

* Mann-Whitney test 
Table 3. Quality indicators during years 2007 and 2009

\begin{tabular}{llll}
\hline & $\mathbf{2 0 0 7}$ & $\mathbf{2 0 0 9}$ & $\boldsymbol{p}$ \\
\hline Readmissions Rate & $7.3 \%$ & $7.4 \%$ & $>.05$ \\
Risk-adjusted Readmissions Rate $^{*}$ & 1.07 & 1.04 & $>.05$ \\
Complications Rate $^{*}$ & $5.2 \%$ & $6.8 \%$ & $>.05$ \\
Risk-adjusted Complications Rate $^{*}$ & 0.96 & 1.16 & $>.05$ \\
Mortality Rate $_{\text {Risk-adjusted Mortality Rate }}^{*}$ & $5.1 \%$ & $4.6 \%$ & $<.05$ \\
\hline
\end{tabular}

${ }^{*}$ Comparison group created from peer hospitals, data from years 2009 and $2010^{[17]}$.

\section{Discussion}

The transformation of hospital regular wards into short-stay wards allowed an increase in patient turnover per bed, a reduction in the number of surgery cancellations due to lack of beds and a reduction in costs due to closing these wards at weekends and therefore the need of less staff. Advances in medical knowledge increasingly allow that every time more diagnostic and therapeutic techniques become possible while, at the same time, demand increases. However, the need of both a higher specialization of the clinical practice and a higher volume of activity determines that a lot of healthcare systems face difficulties to cope with the organization and the management of their healthcare facilities ${ }^{[7,9]}$.

The challenge is to achieve sustainability between treating people individually and specifically, while also using methods of mass production to provide equal service with limited resources. It is clear that health care is too broad and diverse to be treated as a single industry with a single perspective and an only solution to manage its operations. However, Complexity Theory holds that if all the elements are viewed through a conceptual lens, apparent variety can reveal meaningful, even simple, patterns. Therefore, health care should be segmented or classified into parts that, in some important aspects, are homogeneous enough to be managed ${ }^{[10]}$. Following this evidence, it is possible to segment healthcare working practices in different parts, enough similar as to be standardized ${ }^{[11]}$. In this sense, SSU allowed to put together patients of different medical specialties with similar expected length of stay and care needs, so that these wards could be closed at weekends and opened again every Monday morning. The main goal of this transformation was to improve performance through designing and running a hospital organization that created high quality care cost-effectively.

It has been described that the lack of focus or homogeneity in management, for example to place in the same production line standard processes with highly specialized processes ${ }^{[12]}$, or to use the same resources for emergency cases as for scheduled cases, creates problems of capacity use and waiting times ${ }^{[13]}$. In this study we proposed a new group among scheduled patients, the short-length of stay ${ }^{[14]}$. In this sense, we observed that there was a large volume of patients staying one night after the implementation of admission "in the same day as surgery" policy. In all situations they were common processes, surgery or a diagnostic or interventionist test, highly standardized and with an expected low complication rate $^{[7]}$. This type of patients were around $60 \%-70 \%$ of the admissions - after excluding Day Surgery - and due to their low complexity and no need of specific nursing care, were usually allocated in a bed out of their expected ward. This situation could lead to a longer length of stay, as nurse supervision of the outlier ward did not know the expected length of stay and medical staff would always focus on more complex and severe patients and leave simple cases for the late morning or even the next day. In this sense, we found sensible to put together all the low complexity patients in specific wards, with nurse staff equally focused on nurse care and patient flow management. In addition, there was the opportunity to plan discharge when planning admission, as the complication rate related to hospitalization was low. This idea helped to increase the number of planned discharges in 2009 compared to the 2007 situation. Moreover, to separate high volume and low complexity patients from the rest of scheduled admissions competing for the same type of beds reduced the number of last moment cancellations due to lack of bed, as more severe, complex and more theatre time consuming cases were usually prioritized over simpler cases. So, there was an opportunity to improve safeness for low complexity patients in the SSU as 
we could plan in advance: patient bed location and bed assignment, nursing care needs and patient discharge. In the past, there had been exclusively clinical prioritization criteria when deciding which patient was admitted and which one was cancelled. There was not any possibility to consider other additional elements such as the surgery theatre and the bed capacity optimization and the revenues for the financial sustainability of the hospital.

The differences between quality indicators of the whole hospital admissions, emergency and scheduled, showed in Table 3 were not significant except for the risk-adjusted mortality rate, which significantly decreased from 1.02 to 0.89 . These changes could be attributed to clinical data management decisions and epidemiologic factors out of the scope of this study as the design and conduction of it is in real terms, uncontrolled, that were added to the impact that could be attributed to the improvements in patient flow as a result of this study.

With this intervention, patients' admission and discharge was quick, agile and following a scheduled protocol, adjusting the time of admission before surgery and of discharge, being the latter a prioritized task early in the morning in order to free up beds. The patient flow improved as it is known that daily bed shortages are mostly influenced by the timing of arrival and discharge of patients with a short length of stay ${ }^{[15]}$. Occasionally, there could be a demand peak and not enough SSU-beds to allocate all the patients. If that was the case, there was the possibility to prepare some short-stay patients in the chairs from the surgery reception unit ${ }^{[16]}$, and locate them in a SSU-bed after the surgery, limiting the need of last moment cancellation to the minimum possible. Besides we did not conduct a survey to test patients' satisfaction with the SSU, we can deduce that the decrease of last moment cancellations indicator had a high impact in terms of improving patients' experience and perceived quality of care.

Another strength of creating a SSU is that there was the possibility of closing these wards at weekends. From a global point of view, that was as if we were putting together all the free beds that would not be needed during the weekend. So, every Friday two hospital wards were closed and that meant a 50\% reduction of the number of nurses and auxiliary nurses needed. This advantage was at the same time the main drawback, as some of the patients of the SSU were moved to a regular ward every Friday. These patients were all Friday admissions and patients that needed more than one night-stay. However, as all admissions had a planned discharge, most patients left the hospital on Saturday and then there were free beds for emergency admissions during the weekend.

\section{Conclusions}

The efficient management of patient flow is important for the benefit of both patients and the hospital. As we have showed, there is an opportunity to redesign patients' pathways in order to improve patient flow and optimize bed management, which ultimately improves quality and the safeness of patient care. That is to say these patients are admitted promptly to an appropriate bed and stay for no longer than is necessary. The SSU was an appropriate solution to cope with bed demand, as it could allocate an important amount of scheduled patients and therefore theatre resources were used properly, the same as with other diagnostic tests and interventionist procedures. Finally, the creation of a SSU promoted a high level of satisfaction among professionals, especially physicians; in fact, the bottleneck to the hospitalists' workflow was not the lack of beds any more.

\section{Conflict of interests}

The authors declare that they have no competing interests.

\section{References}

[1] Green LV. Capacity Planning and Management in Hospitals. In: Brandeau M, Sainfort F, Pierskalla W (eds.) Operations Research and Health Care: A Handbook of Methods and Applications. Springer US. 2005; p: 15-41. 
[2] Kroneman M, Siegers JJ. The effect of hospital bed reduction on the use of beds: a comparative study of 10 European countries. Soc Sci Med. 2004; 59(8): 1731-40. http://dx.doi.org/10.1016/j.socscimed.2004.01.036

[3] Proudlove NC, Gordon K, Boaden R. Can good bed management solve the overcrowding in accident and emergency departments? Emerg Med J. 2003; 20(2): 149-55. http://dx.doi.org/10.1136/emj.20.2.149

[4] Kolker A. Process modelling of emergency department patient flow: effect of patient length of stay on ED diversion. J Med Syst. 2008 Oct; 32(5): 389-401. http://dx.doi.org/10.1007/s10916-008-9144-x

[5] Bekker R, Koeleman PM. Scheduling admissions and reducing variability in bed demand. Health Care Manag Sci. 2011; 14(3): 237-49. http://dx.doi.org/10.1007/s10729-011-9163-x

[6] Vissers JM. Patient flow-based allocation of inpatient resources: A case study. Eur J Oper Res. 1998; 105(2): 356-70. http://dx.doi.org/10.1016/S0377-2217(97)00242-7

[7] Bohmer R. Designing Care: Aligning the Nature and Management of Health Care. Ed Harvard Business Press, 2009.

[8] Ortiga B, Salazar A, Jovell A, Escarrabill J, Marca G, Corbella X. Standardizing admission and discharge processes to improve patient flow: a cross sectional study. BMC Health Serv Res. 2012; 12: 180. http://dx.doi.org/10.1186/1472-6963-12-180

[9] Battistella R.M. Health care turning point: why single payer won’t work, ed The MIT Press, 2010.

[10] Miller, J.H., Page, S.E. Complex adaptive systems: An introduction to computational models of social life. Princeton Univ Pr, 2007.

[11] Lillrank P, Groop PJ, Malmström TJ. Demand and supply-based operating modes—a framework for analyzing health care service production. Milbank Q. 2010 Dec; 88(4): 595-615. http://dx.doi.org/10.1111/j.1468-0009.2010.00613.x

[12] Hayes, R.H., Wheelwright, S.G. The dynamics of process-product life cycles. Harvard Business Review. 1979; 57(2): 127-36.

[13] Joustra, P., Van der Sluis, E., Van Dijk, N.M. To pool or not to pool in hospitals: a theoretical and practical comparison for a radiotherapy outpatient department. Ann Oper Res 2010; 178(1): 77-89. http://dx.doi.org/10.1007/s10479-009-0559-7

[14] Sanderson, H., Mountney, L. The development of patient groupings for more effective management of health care. Eur J Public Health. 1997; 7(2): 210-4. http://dx.doi.org/10.1093/eurpub/7.2.210

[15] Allder, S., Silvester, K., Walley, P. Managing capacity and demand across the patient journey. J R Coll Physicians. 2010; 10(1): 13-5. http://dx.doi.org/10.7861/clinmedicine.10-1-13

[16] Ortiga B, Capdevila C, Salazar A, Viso MF, Bartolomé C, Corbella X. Effectiveness of a Surgery Admission Unit for patients undergoing major elective surgery in a tertiary university hospital. BMC Health Serv Res. 2010; 10: 23.

http://dx.doi.org/10.1186/1472-6963-10-23

[17] Hospitales Top 20 Metodología: Hospitales Top 20 Metodología. Iasist. [Internet] 2012. Available from: http://www.iasist.com/files/MetodologiaResultadosTOP_2012_0.pdf (22 August 2013, date last accessed) 